\title{
Implementing Digital Imaging in the Developing World
}

\section{J. Raymond Geis ${ }^{1,2}$}

Received: 6 August 2020 / Revised: 6 August 2020 / Accepted: 13 August 2020 / Published online: 8 September 2020

(C) Society for Imaging Informatics in Medicine 2020

In this issue, Elahi et al. present an enlightening article on the challenges they encountered installing PACS in a low resource environment at University College Hospital (UCH), Ibadan, Nigeria.

Terms like "Global Health" and "Developing Countries" are ill-defined and may not provide useful description of settings such as Elahi et al. encountered. More useful terms include "heterogeneously industrialized regions" or the one they use, "low resource environment." These terms apply not only to places like Nigeria but also to varying extent even to locales in the USA and other "Developed Countries." Low resource environments may exist anywhere with sufficient levels of poverty and/or corruption.

Digital medical imaging and PACS are tech-resourceheavy processes that require every facet to be reliable: digital imaging equipment, computer hardware and software, digital communication infrastructure, and strong cybersecurity. They require people with appropriate domain-specific education to be involved at all stages throughout their lifecycles. At a more basic level, they require stable electricity, a dependable source of spare parts and repair infrastructure, and a level of general security and lack of corruption such that every component remains reliable.

Digital medical imaging has potential to improve healthcare in low resource environments. Beyond this, the ethical goal is ultimately to transfer not only just technology but also skills and value to these areas. To make a perpetual contribution to development, digital imaging and PACS should provide not only better healthcare but they must also generate more higher-paying local jobs. These settings should not only simply consume digital imaging technology but also participate in producing it.

J. Raymond Geis

1 Department of Radiology, National Jewish Health, Denver, CO, USA

2 Fort Collins, USA
SIIM has a vital role to play here. As a primary source of education in all facets of imaging informatics, SIIM has the ability to provide the domain-specific education needed throughout the PACS lifecycle. For this education to be most effective requires understanding both the domain, and also cultural awareness of who is learning, and the environments in which they are learning. In these settings, learners will start with heterogeneous skill sets, in some areas exceptionally upto-date, and other areas where they have unexpectedly significant gaps. One of SIIM challenges is to develop assessment tools to identify skills and gaps. Such assessment will help SIIM focus its education appropriately. SIIM will also continue to work with others to identify and promote existing education and support resources.

The value chain for these informatics professionals is based both on their skills and on documenting their education. SIIM will want to work with informatics professionals in these settings in order to define the most useful approaches to testing and certification. While current CIIP certification will be warranted in many situations, other certificates for specific subsections or skill levels may be desirable.

Elahi et al. provide instructive examples of how they solved local technical issues during PACS planning and implementation. They resolved what many of us would see as entirely unexpected and daunting barriers, and they help lead the way as digital imaging, PACS, and imaging informatics extend throughout the world.

Publisher's Note Springer Nature remains neutral with regard to jurisdictional claims in published maps and institutional affiliations. 